\title{
Sublocalization of Rab23, a mediator of Sonic hedgehog signaling pathway, in hepatocellular carcinoma cell lines
}

\author{
HAI-JI SUN ${ }^{1,3}$, YUN-JIAN LIU ${ }^{2}$, NA LI $^{1}$, ZHI-YONG SUN ${ }^{2}$, HAI-WANG ZHAO ${ }^{2}$, CHEN WANG $^{2}$, HUI LI $^{2}$, \\ FANG-MING MA ${ }^{2}$, SONG-MEI SHI ${ }^{2}$, XIAO-QIU XU ${ }^{2}$, ZHE-YU CHEN $^{1}$ and SHU-HONG HUANG ${ }^{1}$ \\ ${ }^{1}$ Department of Neurobiology, Shandong Provincial Key Laboratory of Mental Disorders, School of Medicine, \\ Shandong University, Jinan; ${ }^{2}$ Department of Hepatobiliary Surgery, Municipal Hospital of Heze, Heze; \\ ${ }^{3}$ College of Life Science, Shandong Normal University, Jinan, Shandong, P.R. China
}

Received June 3, 2012; Accepted September 12, 2012

DOI: $10.3892 / \mathrm{mmr} .2012 .1094$

\begin{abstract}
The novel member of the Rab family of GTPases, Rab23, is an essential negative regulator of the Sonic hedgehog (Shh) signaling pathway. Loss of function mutation of the Rab23 gene causes abnormal development of the neural tube in mice and in certain human congenital diseases. The aberrant overexpression of Rab23 has been associated with various diseases, such as gastric, hepatocellular and lung cancer. The exact function of Rab23 in hepatocellular carcinomas (HCCs), however, remains unknown. Previously, we reported the abnormal sublocalization of Rab23 in lung cancers. In the current study, we investigated the role of Rab23 in HCCs. We report the distinct sublocalization pattern of Rab23 in HCC cell lines. This difference depends on the GDP/GTP-binding form, and inhibition of the Rab23 cycle decreases the expression and nuclear localization of Gli1.
\end{abstract}

\section{Introduction}

The small GTPases of the Rab family play a central role in the regulation of vesicular trafficking through interaction with an extensive range of protein partners. Rabs comprise the largest subfamily of Ras-like GTPases, and in mammals at least 60 members have been identified, but the majority of them have not been studied at the functional level (1-7).

As a novel member of the Rab family of GTPases, Rab23, has recently been found to play a role in vesicular trafficking

Correspondence to: Dr Yun-Jian Liu, Department of Hepatobiliary Surgery, Municipal Hospital of Heze, 2888 Caozhou Road, Heze, Shandong, P.R. China

E-mail: liuyunjiand1234@126.com

Dr Shu-Hong Huang, Department of Neurobiology, Shandong Provincial Key Laboratory of Mental Disorders, School of Medicine, Shandong University, 44 Wenhuaxi Road, Jinan, Shandong 250012, P.R. China

E-mail: shuhonghuang@sdu.edu.cn

Key words: Rab23, hepatocellular carcinoma, Sonic hedgehog pathway and protein transport in eukaryotic cells. Rab23 is also an essential negative regulator of the Sonic hedgehog (Shh) signaling pathway (8-14). Loss of function mutation of the Rab23 gene causes abnormal development of the neural tube in mice and in certain human congenital diseases $(6,15-19)$. The aberrant overexpression of Rab23 has been associated with various diseases such as gastric, hepatocellular and lung cancer (19-21). The Rab23 homolog in Trypanosoma brucei, TbRab23, is reported to be a nuclear-associated protein, and its function may be to provide stability to the nuclear structure (22). Recently, Chi et al indicated that Rab23 negatively regulates Gli1 activity in a $\mathrm{Su}(\mathrm{Fu})$-dependent manner (23). Rab23 is reported to play crucial roles in autophagy of Group A streptococcus (GAS) (24). We have previously shown the overexpression and abnormal sublocalization of Rab23 in lung cancers (21). Rab23 is expressed in hepatocellular carcinomas (HCCs) and its elevated expression is associated with the development of HCC in Hep3B cells (19). However, the exact pathophysiological roles of $\mathrm{Rab} 23$ in $\mathrm{HCC}$ remain unknown. In the current study, we investigated the role of Rab23 in HCC.

\section{Materials and methods}

Cell culture and plasmid constructs. Human hepatoma Hep3B and HepG2 cell lines were obtained from the American Type Culture Collection (Rockville, MD, USA) and routinely maintained in Dulbecco's modified Eagle's medium, supplemented with $10 \%$ fetal bovine serum. For gene overexpression, constructs expressing human Rab23 were prepared as previously described (21). Myc-tagged human Rab23 and its mutants (Rab23-SN and Rab23-QL) were subcloned into a pCDNA3.1 expression plasmid (Invitrogen, Carlsbad, CA, USA). All the mutants were generated by two-step PCR. All the constructs were confirmed by DNA sequencing, in order to exclude potential PCR-introduced mutations. The study was approved by the ethics committee of Shandong University.

For gene knockdown, the pSuper-RFP vectors (OligoEngine, Seattle, WA, USA) were used to transcribe functional small interfering RNA (siRNA). In the vectors, oligonucleotides targeting different genes were inserted downstream of the $\mathrm{H} 1$ promoter, with their veracity confirmed 
by double digestion and sequencing. The target sequences for human Rab23 were CCAGAACTAACGCATTCAA. The expression levels of Rab23 proteins in Hep3B transfected with the resulting siRNA or the scramble siRNA were analyzed by immunoblotting with individual antibodies.

Reagents and antibodies. Antibodies were purchased as follows: goat anti-Rab23 antibodies and mouse anti-tubulin antibodies from Sigma-Aldrich (St. Louis, MO, USA); mouse anti c-Myc and rabbit anti-Gli1 from Santa Cruz Biotechnology Inc. (Santa Cruz, CA, USA); Alexa Fluor 488or 594-conjugated goat anti-mouse or rabbit $\operatorname{IgG}(\mathrm{H}+\mathrm{L})$ from Invitrogen; and horseradish peroxidase (HRP)-conjugated goat anti-mouse or rabbit $\mathrm{IgG}$ antibodies from Calbiochem (La Jolla, CA, USA). The restriction enzymes were purchased from Fermentas (Hanover, MD, USA). Vectashield mounting medium was obtained from Vector Laboratories (Burlingame, CA, USA). The other reagents were from Sigma-Aldrich.

Immunofluorescence analysis. Hep3B or HepG2 cells were transfected using Lipofectamine ${ }^{\mathrm{TM}} 2000$ transfection reagent according to the manufacturer's instructions. All experiments were performed $48 \mathrm{~h}$ after transfection. Firstly, cells were fixed with $4 \%$ paraformaldehyde in phosphate-buffered saline (PBS) for $10 \mathrm{~min}$. Cultures were washed with PBS three times, and then permeabilized with $0.4 \%$ Triton $\mathrm{X}-100$ in PBS for $10 \mathrm{~min}$. After washing in PBS again, the cells were incubated with blocking solution (PBS containing 10\% normal goat serum) for $1 \mathrm{~h}$ at room temperature. Following incubation with the primary antibodies at $4^{\circ} \mathrm{C}$ overnight, neurons were washed with PBS and incubated with fluorescent secondary antibodies conjugated to Alexa Fluor 488, Alexa Fluor 594 or Cy5 for $1 \mathrm{~h}$ at room temperature. All the immunostained cells were observed with a Zeiss LSM710 confocal microscope (Carl Zeiss, Thornwood, NY, USA), and quantification of labeling intensities was carried out using MetaMorph Software (Universal Imaging Corporation, West Chester, PA, USA).

Statistical analysis. Statistical significance was assessed using Student's t-test or analysis of variance (ANOVA) followed by post-hoc tests. Data were presented as the means \pm SEM, and $\mathrm{p}<0.05$ was considered to indicate a statistically significant difference.

\section{Results}

Different expression pattern of Rab23 in Hep3B and HepG2 cells. In a subset of lung cancers, Rab23 was localized in the nucleus (21). In order to assess whether the nuclear localization of Rab23 also existed in HCC, the expression of Rab23 in the HCC cell lines Hep3B and HepG2 was analyzed. It has been reported that Hep3B has endogenous Rab23 expression (19). Consistent with Liu et al, we also found endogenous Rab23 expression in HepG2 cells (Fig. 1A). The subcellular expression pattern of Rab23 in those two cell lines was investigated. Firstly, immunocytochemical staining was performed to detect the epigenetic Rab23 localization. The cells were transiently transfected with myc-tagged Rab23 expression vectors. Notably, myc-tagged Rab23 protein was located in the nucleus of HepG2 cells, but not in Hep3B cells (Fig. 1B). To confirm the immunocytochemistry results, cell fractionation experiments were performed in Hep3B and HepG2 cells to substantiate the immunofluorescence results. Rab23 appears in the nuclear fraction of HepG2 cells, but not Hep3B cells (Fig. 1C). The results indicate that Rab23 has a different expression pattern in diverse HCC cells.

Difference between HepG2 and Hep3B cells depends on the GDP/GTP binding form. As with other GTPases, Rabs cycle between GDP-bound (off) and GTP-bound (on) forms. The number of known inherited disorders of vesicle trafficking due to Rab cycle defects has increased substantially during the past decade (25). To investigate whether the expression pattern distinction depends on its GDP/GTP binding form,two mutants of Rab23, GDP-binding block form Rab23 S23N and GTP-binding block form Rab23 Q68L, were constructed according to a previous report (11) (Fig. 2A). Immunocytochemical staining was then performed to detect the subcellular localization of the transfected Rab23 mutants in Hep3B cells. As shown in Fig. 2B, Rab23 S23N was detected mostly co-localized with DAPI staining. By contrast, Rab23 Q68L showed mainly cytoplasmic localization. The data suggest that Rab23 localized in the nucleus may be a GDP-binding form, which also suggests that in Hep3B cells the main form of Rab23 was GDP-binding, but in HepG2 the main form of Rab23 was GTP-binding. The Rab23 cycle between two nucleotide-bound states may play a role in the nuclear transport.

Rab23 is involved in the Glil expression pattern. It is well-known that Rab23 works as an essential negative regulator of the Shh signaling pathway, but little is known about its exact function (10-12). The zinc finger transcription factors Gli1 are considered mediators of the Shh signaling pathway (26-29). One report indicates that certain $\mathrm{Su}(\mathrm{Fu})$ mutants that are unable to export Gli from the nucleus to the cytoplasm result in the activation of Shh signaling (30). Recently, Chi et al indicated that Rab23 negatively regulates Gli1 activity in a $\mathrm{Su}(\mathrm{Fu})$-dependent manner (23). To determine whether Rab23 was capable of modulating the Shh activity by controlling the transporting of Gli from the nucleus to the cytoplasm, Rab23 Q68L was transfected into Hep3B cells, and the subcellular localization of Gli1 was detected by immunocytochemical staining. As shown in Fig. 3A, the Rab23 GTP-binding mutant reduces the nuclear localization of Gli1. Further, western blotting showed that transfection of the Rab23 Q78L mutant causes a decrease in Gli1 protein of $60 \pm 3 \%$ (p<0.05; Fig. 3B). These results indicate that Rab23 affects the Gli1 expression pattern and expression level.

Rab23 expression is not affected by Shh signaling pathway activity. The abnormal expression of Rab23 in HCCs has been previously reported (19), and the results of the current study indicate that Rab23 may play roles in the transport of Gli1. It has been reported that many important regulators of the Shh signaling pathway were the target genes of Gli1 such as Patched, Gli1 itself and HIP. To investigate whether Rab23 is also a target gene of Gli1; in other words, if the abnormal expression of Rab23 is caused by Shh signaling pathway activity; we treated the Hep3B cells with KAAD-cyclopamine (a specific inhibitor of Shh intracellular signaling) to inhibit 

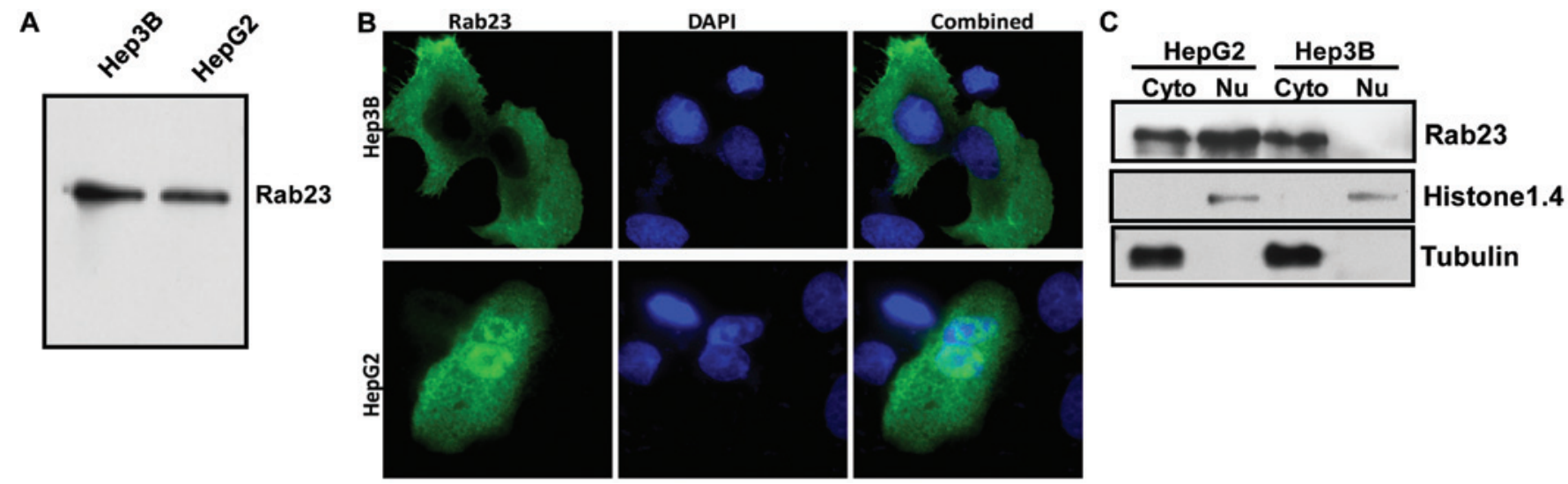

Figure 1. Rab23 is localized in the nuclei of HepG2 cells, but not of Hep3B cells. (A) Western blot analysis of Rab23 protein in HepG2 and Hep3B cells. (B) HepG2 and Hep3B cells were transfected with Rab23 vectors (tagged with myc). Cells were fixed and stained for myc-containing proteins and the nuclei were stained with DAPI. Cells were then analyzed by confocal microscopy. (C) Endogenous Rab23 protein expression in different cell compartments was detected in HepG2 and Hep3B cells by western blotting following cell fractionation. Histone1.4 was used as a marker for nuclear fraction whereas tubulin was used as a cytoplasmic protein marker.

A GDP- binding block
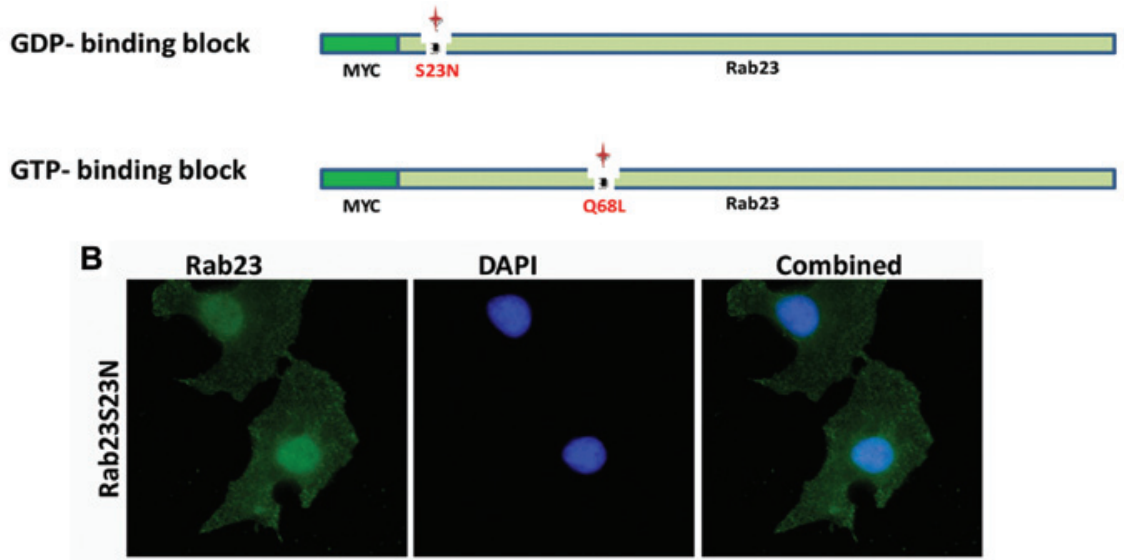

DAPI

Combined
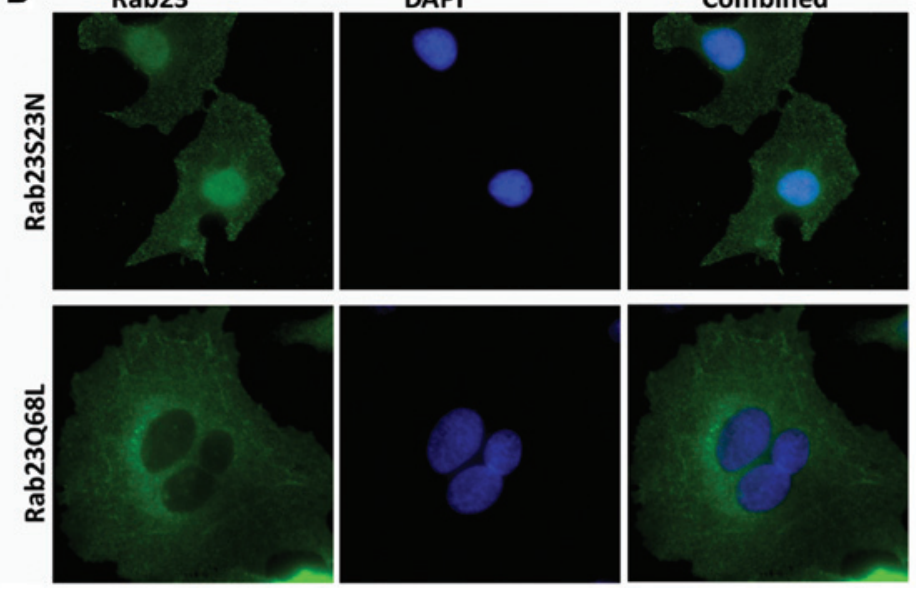

Figure 2. The GDP-binding form of Rab23 is localized in the nuclei. (A) Schematic representation of human Rab23 GDP or GTP binding block mutants. (B) HepG2 and Hep3B cells were transfected with Rab23 mutants (tagged with myc). Cells were fixed and stained for myc-containing proteins and the nuclei were stained with DAPI. Cells were then analyzed by confocal microscopy.
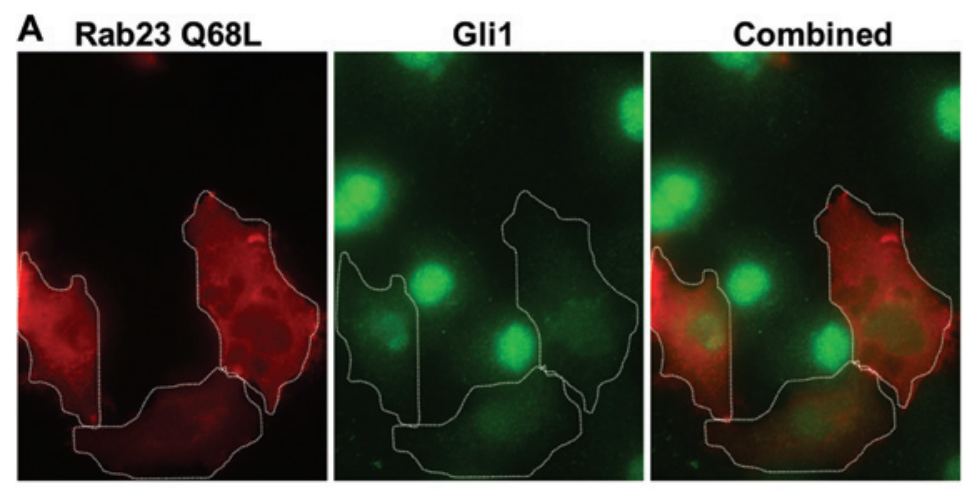

B

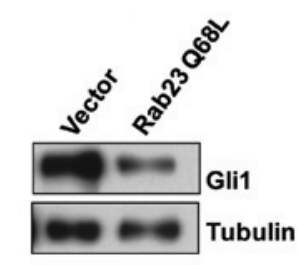

Figure 3. Rab23 is involved in the control of Gli1 localization. (A) Hep3B cells were transfected with Rab23 Q68L. Cells were fixed and stained for myc-containing proteins (red) and Gli1 (green). (B) Extracts from Hep3B cells transfected with Rab23 Q68L or empty vector were subjected to western blotting and probed with antibodies to Rab23 or $\beta$-tubulin (loading control). 
A

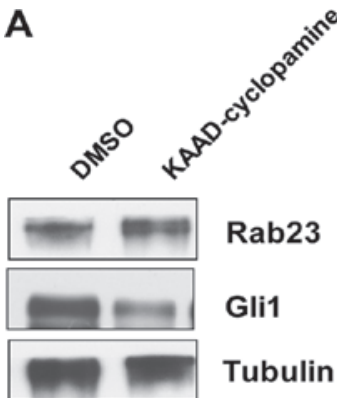

B

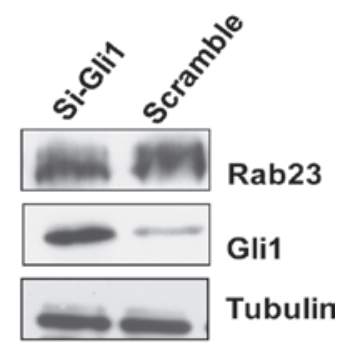

Figure 4. Rab23 expression is not affected by Sonic hedgehog (Shh) signaling pathway activity. (A) Extracts from Hep3B cells transfected with si-Gli1 were subjected to western blotting and probed with antibodies to Rab23, Gli1 or $\beta$-tubulin (loading control). (B) Extracts from Hep3B cells treated with Shh signaling pathway inhibitor KAAD-cyclopamine were subjected to western blotting and probed with antibodies to Rab23, Gli1 or $\beta$-tubulin.

the pathway activity and to detect whether this treatment is capable of downregulating Rab23 expression. Using western blotting, we found that the expression level of Rab23 protein was not changed by KAAD-cyclopamine. Additionally, siRNA knockdown of Gli1 had no effect on the Rab23 expression level. Thus, we determined that the Rab23 expression is not affected by the Shh signaling pathway activity (Fig. 4).

\section{Discussion}

Rab23 is the product of gene open brain (opb), which was first identified in 1994 as a natural mouse mutation (14). Reports have shown that Rab23 acts as a negative regulator of the Shh signaling pathway (9-12). Even as a Rab protein, Rab23 is not previously known to have roles in any established intracellular-trafficking pathways (31). However, in view of the general function of Rab GTPases in vesicular membrane transport, it is conceivable that Rab23 may regulate the cellular dynamics of components of the Shh pathway $(6,7)$. The subcellular distribution pattern of neither Patched nor Smoothened, however, was altered by overexpression of the wild-type, or the GTP or GDP-restricted mutants of Rab23 (11). These results indicate that Rab23 does not directly regulate cellular dynamics of the above proteins, which lie upstream in the Shh signaling pathway. Rab23 may modulate the activities of the Gli transcription factors in more direct ways than previously anticipated, perhaps by directly affecting either their nucleocytoplasmic trafficking or that of relevant components of Shh signaling (31). The Rab23 homolog in Trypanosoma brucei, TbRab23, is reported to be a nuclear-associated protein, and its function may be to provide stability to the nuclear structure (22). Recently, Chi et al indicated that Rab23 negatively regulates Gli1 activity in a $\mathrm{Su}(\mathrm{Fu})$-dependent manner (23).

The abnormal expression of Rab23 in HCCs and the nuclear localization of Rab23 in lung cancers have also been reported $(19,21)$. This study provides three new insights into the sublocalization of Rab23 in HCC cell lines, and its role in regulating Gli1 localization and expression level.

Firstly, Rab23 has different expression patterns in diverse HCC cells. It is important to identify the role of abnormal expression of Rab23 in HCCs to improve diagnosis and treatment; however, it has been little investigated in previous

studies. To better understand the expression profile of Rab23 in HCCs, the expression of transfected Rab23 in two HCC cell lines, Hep3B and HepG2, was investigated. The cells have been reported to have a distinct character (p53 or Shh signaling pathway) (32). The results indicate that Rab23 is capable of localizing in the nucleus of HepG2 cells, but not in the nucleus of Hep3B cells. The various expression patterns of Rab23 in HCCs indicate that Rab23 may function though different mechanisms in different types or subtypes of cancer.

Secondly, evidence suggests that the nuclear localization of Rab23 depends on its GDP/GTP-binding form. Rabs function primarily as molecular switches by controlled hydrolysis of GTP; conformational changes between the GTP- and GDP-bound states facilitate a cycle whereby Rabs bind to discrete internal compartments, providing a role in generating specificity in intracellular vesicular trafficking. The results demonstrate that the GDP-bound form of Rab23 (Rab23 S23N), but not the constitutively active Rab23 form (Rab23 Q68L), was capable of localizing in the nucleus of Hep3B. The difference indicates that the GDP-bound form of Rab23 was the main form existing in HepG2, the GTP-bound form of Rab23 was the main form existing in Hep3B, and also indicates that the GTP- and GDP-bound forms may be important in the function of Rab23. In addition, inhibition of the Rab23 cycle will decrease the expression and nuclear localization of Gli1. This result will help to clarify the exact function of Rab23 in the Shh signaling pathway.

Thirdly, Rab23 expression is independent of Shh activity. Rab23 regulates the Shh signaling pathway by antagonizing the action of Gli1, but Rab23 itself is not the transcriptional target of Shh signaling.

In all, these studies have identified the abnormal Rab23 expression pattern in diverse HCCs, and that Rab23 function may depend on its GDP/GTP-binding form. Since it is still not known why Rab23 expression is different in diverse HCC cells, further studies will be required to identify additional details about this association.

\section{Acknowledgements}

This research was supported by grants from the Doctor Foundation of Heze Municipal Hospital (0012009), and the National Natural Science Foundation of China (30900717).

\section{References}

1. Armstrong J: How do Rab proteins function in membrane traffic? Int J Biochem Cell Biol 32: 303-307, 2000.

2. Bohdanowicz M and Grinstein S: Vesicular traffic: a Rab SANDwich. Curr Biol 20: R311-R314, 2010.

3. Fukuda M: Regulation of secretory vesicle traffic by Rab small GTPases. Cell Mol Life Sci 65: 2801-2813, 2008.

4. Hutagalung AH and Novick PJ: Role of Rab GTPases in membrane traffic and cell physiology. Physiol Rev 91: 119-149, 2011.

5. Olkkonen VM and Stenmark H: Role of Rab GTPases in membrane traffic. Int Rev Cytol 176: 1-85, 1997.

6. Seabra MC, Mules EH and Hume AN: Rab GTPases, intracellular traffic and disease. Trends Mol Med 8: 23-30, 2002.

7. Stenmark H: Rab GTPases as coordinators of vesicle traffic. Nat Rev Mol Cell Biol 10: 513-525, 2009.

8. Boehlke C, Bashkurov M, Buescher A, Krick T, John AK, Nitschke R, Walz G and Kuehn EW: Differential role of Rab proteins in ciliary trafficking: Rab23 regulates smoothened levels. J Cell Sci 123: 1460-1467, 2010. 
9. Eggenschwiler JT, Bulgakov OV, Qin J, Li T and Anderson KV: Mouse Rab23 regulates hedgehog signaling from smoothened to Gli proteins. Dev Biol 290: 1-12, 2006.

10. Eggenschwiler JT, Espinoza E and Anderson KV: Rab23 is an essential negative regulator of the mouse Sonic hedgehog signalling pathway. Nature 412: 194-198, 2001.

11. Evans TM, Ferguson C, Wainwright BJ, Parton RG and Wicking C: Rab23, a negative regulator of hedgehog signaling, localizes to the plasma membrane and the endocytic pathway. Traffic 4: 869-884, 2003.

12. Evans TM, Simpson F, Parton RG and Wicking C: Characterization of Rab23, a negative regulator of sonic hedgehog signaling. Methods Enzymol 403: 759-777, 2005.

13. Marcos I, Borrego S and Antiñolo G: Molecular cloning and characterization of human RAB23, a member of the group of Rab GTPases. Int J Mol Med 12: 983-987, 2003.

14. Olkkonen VM, Peterson JR, Dupree P, Lütcke A, Zerial M and Simons K: Isolation of a mouse cDNA encoding Rab23, a small novel GTPase expressed predominantly in the brain. Gene 138: 207-211, 1994

15. Alessandri JL, Dagoneau N, Laville JM, Baruteau J, Hébert JC and Cormier-Daire V: RAB23 mutation in a large family from Comoros Islands with Carpenter syndrome. Am J Med Genet A 152A: 982-986, 2010

16. Guo A, Wang T, Ng EL, Aulia S, Chong KH, Teng FY, Wang Y and Tang BL: Open brain gene product Rab23: expression pattern in the adult mouse brain and functional characterization. J Neurosci Res 83: 1118-1127, 2006.

17. Jenkins D, Baynam G, De Catte L, Elcioglu N, Gabbett MT, Hudgins L, Hurst JA, Jehee FS, Oley C and Wilkie AO Carpenter syndrome: extended RAB23 mutation spectrum and analysis of nonsense-mediated mRNA decay. Hum Mutat 32: E2069-E2078, 2011.

18. Jenkins D, Seelow D, Jehee FS, et al: RAB23 mutations in Carpenter syndrome imply an unexpected role for hedgehog signaling in cranial-suture development and obesity. Am J Hum Genet 80: 1162-1170, 2007.

19. Liu YJ, Wang Q, Li W, Huang XH,Zhen MC, Huang SH, Chen LZ Xue L and Zhang HW: Rab23 is a potential biological target for treating hepatocellular carcinoma. World J Gastroenterol 13: 1010-1017, 2007.

20. Hou Q, Wu YH, Grabsch H, et al: Integrative genomics identifies RAB23 as an invasion mediator gene in diffuse-type gastric cancer. Cancer Res 68: 4623-4630, 2008.
21. Huang S, Yang L, An Y, Ma X, Zhang C, Xie G, Chen ZY, Xie J and Zhang $\mathrm{H}$ : Expression of hedgehog signaling molecules in lung cancer. Acta Histochem 113: 564-569, 2011.

22. Dhir V and Field MC: TbRAB23; a nuclear-associated Rab protein from Trypanosoma brucei. Mol Biochem Parasitol 136: 297-301, 2004

23. Chi S, Xie G, Liu H, Chen K, Zhang X, Li C and Xie J: Rab23 negatively regulates Glil transcriptional factor in a $\mathrm{Su}(\mathrm{Fu})$ dependent manner. Cell Signal 24: 1222-1228, 2012.

24. Nozawa T, Aikawa C, Goda A, Maruyama F, Hamada S and Nakagawa I: The small GTPases Rab9A and Rab23 function at distinct steps in autophagy during Group A Streptococcus infection. Cell Microbiol 14: 1149-1165, 2012.

25. Corbeel L and Freson K: Rab proteins and Rab-associated proteins: major actors in the mechanism of protein-trafficking disorders. Eur J Pediatr 167: 723-729, 2008.

26. Bian YH, Huang SH, Yang L, Ma XL, Xie JW and Zhang HW: Sonic hedgehog-Gli1 pathway in colorectal adenocarcinomas. World J Gastroenterol 13: 1659-1665, 2007.

27. Chen G, Goto Y, Sakamoto R, Tanaka K, Matsubara E, Nakamura M, Zheng H, Lu J, Takayanagi R and Nomura M: GLI1, a crucial mediator of sonic hedgehog signaling in prostate cancer, functions as a negative modulator for androgen receptor. Biochem Biophys Res Commun 404: 809-815, 2011.

28. Lee J, Platt KA, Censullo P and Ruiz i Altaba A: Gli1 is a target of Sonic hedgehog that induces ventral neural tube development. Development 124: 2537-2552, 1997.

29. Rong ZX, Fang CH,Zhu DJ and Liu SJ: Expression of Smo protein and the downstream transcription factor Glil protein in Sonic hedgehog signal transduction pathway in gastric carcinoma. Nan Fang Yi Ke Da Xue Xue Bao 26: 1728-1731, 2006 (In Chinese).

30. Tukachinsky H, Lopez LV and Salic A: A mechanism for vertebrate Hedgehog signaling: recruitment to cilia and dissociation of SuFu-Gli protein complexes. J Cell Biol 191: 415-428, 2010.

31. Wang Y, Ng EL and Tang BL: Rab23: what exactly does it traffic? Traffic 7: 746-750, 2006.

32. Huang S, He J, Zhang X, et al: Activation of the hedgehog pathway in human hepatocellular carcinomas. Carcinogenesis 27: 1334-1340, 2006 